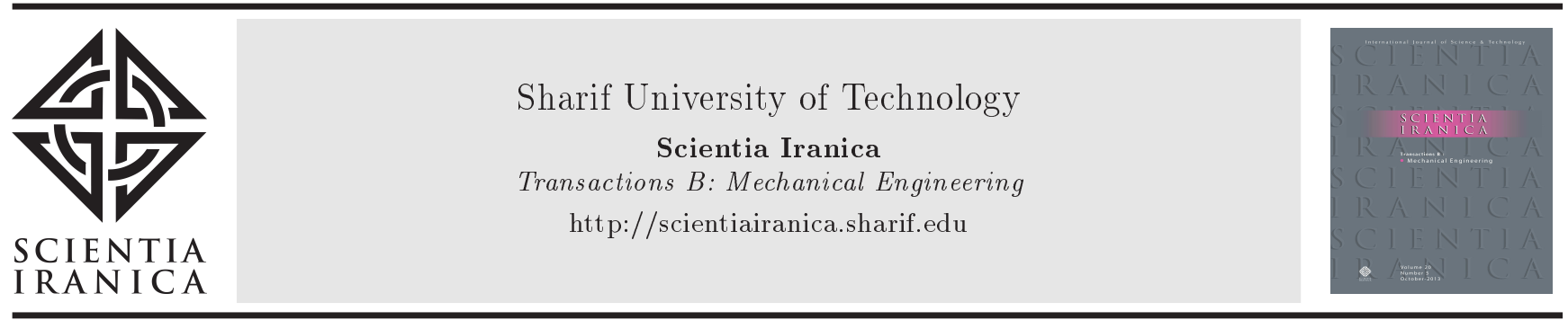

\title{
Development of a new mechanism to change velocity in a helical swimmer robot at low Reynolds number
}

\author{
H. Sayyaadi* and Sh. Bahmanyar \\ School of Mechanical Engineering, Center of Excellence in Hydrodynamics and Dynamics of Marine Vehicles, Sharif University of \\ Technology, Tehran, Iran.
}

Received 6 December 2016; received in revised form 3 September 2017; accepted 6 June 2018

\author{
KEYWORDS \\ Biomimetic helical \\ swimmer; \\ Double Helices \\ Propulsion \\ Mechanisms (DHPM); \\ Shape Memory Alloy \\ (SMA); \\ Propulsive helix \\ (outer helix); \\ SMA helix (inner \\ helix); \\ Variable forward \\ velocity.
}

\begin{abstract}
This paper presents a new mechanism with unique and prominent features for a helical swimmer robot. "Double Helices Propulsion Mechanism" consists of two parallel helices with a single axis rotating in the same direction. The outer helix acts as the main propulsion component, and the inner helix, which is made of a Shape Memory Alloy (SMA), controls the forward velocity during swimming. This mechanism, by varying the geometrical parameters of its helical tail, can change the forward velocity of the helical swimmer robot that is required by its predefined missions. In order to study the effects of geometric parameters on the forward velocity in the single helical swimmer, a hydrodynamic model based on Slender Body Theory (SBT) is implemented. Moreover, in order to validate the predicted results, a scaled-up macro-dimension prototype with a single helical tail and Reynolds number of less than one is built. Finally, the performance of the double helices system is estimated by modelling the dynamics of the motion in different tail lengths. This comparison indicates that this mechanism increases the forward velocity and efficiency of swimmer robot; it can produce variable forward velocities at each frequency.
\end{abstract}

(C) 2018 Sharif University of Technology. All rights reserved.

\section{Introduction}

Swimmer robots whose movements are inspired from the locomotion mechanism of microorganisms can be used as effective devices in the future for various medical and industrial applications and become suitable for predefined missions; therefore, it is essential to investigate their performances and the ability to move in different directions. Based on their microor macro-size dimensions, different applications could be defined for swimmer robots. In terms of medical applications, some of the applications defined for micro-sized swimmer robots include delivering drugs to

*. Corresponding author. Tel.: +982166165682 E-mail addresses: sayyaadi@sharif.edu (H. Sayyaadi); bahmanyar@mech.sharif.edu (Sh.Bahmanyar)

doi: $10.24200 /$ sci.2018.20031 target regions or taking images of damaged areas [1-3]. Furthermore, with regard to industrial missions, some of the applications defined for macro-sized swimmers include the inspection and imaging of pipes that carry highly viscous fluids. In view of different applications considered for this type of swimmer robots, mobility and maneuverability are important factors in future therapeutic medical and industrial applications. In this respect, at low Reynolds conditions, microorganisms with helical tails are considered as efficient swimmers in propulsion [4,5], and many researchers have tried to carry out more extensive researches on these microorganisms and extract important hydrodynamic parameters, such as forward velocity, thrust force, and their efficiency during swimming [6,7], and to design swimmer robots in micro or macro dimensions through drawing inspiration from them.

According to the theoretical and experimental researches $[3,7-10]$, the important geometrical parame- 
ters with significant effects on swimmer velocity and efficiency in unbounded fluid or bounded fluid are the length and amplitude of helical tail. In these researches, Edd et al. [3] explored the effects of the synthetic flagellum geometry (pitch angle and amplitude of helical tail) and frequency of rotation on efficiency and swimming speed. By using RFT method, Behkam et al. [8] compared the performances of various helical tail, and showed that force, torque, velocity, and efficiency of such swimmers are functions of geometry only, and velocity and efficiency will develop by increasing the number of waves of helical tails. By applying the Design Of Experiments (DOE) method, Xu et al. [9] investigated the effects of the geometrical parameters of a magnetic swimmer robot's tail on the swimmer's performance by actuating external filed under low Reynolds conditions. They demonstrated that the most effective geometrical parameter is the pitch angle, and the swimmer robot exhibits a better performance as the pitch angle increases. Temel et al. [10] used a three-dimensional CFD model of robots with cylindrical bodies and helical tails inside circular channels and investigated the effects of the radial position of the swimmer, length and radius of the cylindrical body, number and amplitude of helical waves on the tail on the linear and angular velocities of the swimmer. They concluded that the most effective parameter in forward velocity is the number of waves. Different theories have been presented for exploring the relationship between the forward velocity of a swimmer and the geometrical parameters of its helical tail [11-18]. One of the wellknown theories is the theory of Gray and Hancock [11], considered as the pioneers of the Resistive Force Theory (RFT) and Slender Body Theory (SBT). Subsequently, Lighthill [12] improved the SBT. Through considering the fluid flow around the swimmer body and the bodyfluid interaction, he estimated the forward velocity of a swimmer robot based on its geometrical parameters.

To the best of authors' knowledge, in this article, there is no research investigating a mechanism which can change the forward velocity of the swimmer based on varying the geometrical parameters of its helical tail during swimming.

In our research, attempt is made to clarify that the wavelength and amplitude of the helical tail change simultaneously by varying the helical length. Furthermore, the actual length of the helical tail and the number of waves remain constant. Then, in order to study the effects of geometric parameters on the forward velocity in the single helical swimmer by changing the helical length, a hydrodynamic model based on Slender Body Theory (SBT) is implemented. In order to validate the predicted results, a scaledup macro-dimension prototype with a single helical tail and Reynolds number of less than one is built. According to these results, a new mechanism made of SMA material has been designed and proposed which has three main features, not shared by pervious researches:

1. By altering the geometrical parameters of the helical length, the swimmer's forward velocity can change into the desired velocity during swimming with respect to the pre-defined mission for the swimmer;

2. The proposed altering velocity mechanism, also called the Double Helices Propulsion Mechanism (DHPM), consists of an outer helical tail, as the main propulsion system of the swimmer, and an inner helical tail, made of shape memory alloy. These two parallel and coaxial helices rotate in the same direction with similar frequencies;

3. This concept can be used for swimmers with helical propulsion regardless of its size and internal actuation system. Then, the performances of the double helices system, such as forward velocity and efficiency, are estimated through modelling of the viscous drag on the helical swimmer in the single- and double-helix propulsion systems and are compared with the performance of the single helix propulsion system.

This paper can be organized as follows: in Sections 2, the theoretical relations in SBT are used to study the effect of the helical tail's length of the single helix on the swimmer's forward velocity. In Section 3, in order to validate the theoretical result, a scaled-up macro-dimension prototype with a single helical tail is built in Reynolds number of less than one. According to the obtained results in pervious sections, in Section 4, the new propulsion system is introduced. Then, based on the specifications of the helical swimmer robot designed in Section 3 as the main propulsion system, the shape memory alloy helix is proposed and designed as the material of inner helical tail to change the desired length of the entire helix. Then, the way of actuating the inner helical tail (made of shape memory alloy) for achieving a variable forward velocity is investigated. Finally, in Section 5, the hydrodynamic model of the swimmer robot with the double helices propulsion mechanism is presented, and the swimmer performance is evaluated using the RFT; subsequently, the performances of the single- and double-helix propulsion systems are compared.

\section{Theoretical modelling of the single helical swimmer}

The SBT has been used to investigate the effects of the helical tail's length on the forward velocity of the swimmer, while all the other geometrical parameters have changed. The theory of Lighthill [12] is characterized as an effective and practical method for computing 


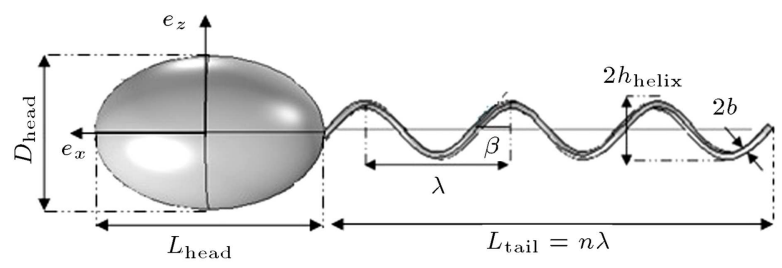

Figure 1. The geometrical parameters of the helical swimmer consisting of an elliptical body and helical tail. The helical tail has a single rotational degree of freedom about the main axis.

the forward velocity $(U)$ of microorganisms that swim by means of a helical flagellum. In this approach, by considering an appropriate distance between swimmer and walls, their effects on the swimmer can be ignored. The other assumptions used in the computations include the slenderness of the helical tail and the ellipsoid shape of swimmer body. To ensure that the slender body approximation is valid, the radius of the crosssection of helical tail should be much smaller than the helical length $(b \ll L)$. Figure 1 shows the geometry of a helical swimmer robot that consists of an elliptical body with a specification of diameter $\left(d_{\text {head }}\right)$ length ( $\left.L_{\text {head }}\right)$, connected to a helical tail via a coupling. The helical tail is assumed to have wave amplitude $\left(2 h_{\text {helix }}\right)$, helix angle $(\beta)$, radius of helix cross-section $(b)$, and wavelength $(\lambda)$ with the number of wavelength $(n)$. The swimmer is propelled forward as the helical tail spins around its main axis, at angular velocity of $\omega$, in a counterclockwise direction.

In this method, the relation between the geometry of helical tail and the swimmer's forward velocity is defined as follows:

$$
\begin{aligned}
\frac{U}{\omega b}= & \left(1+\left(\psi\left(d_{\text {head }} / 2\right) \alpha / n \lambda\right)\right)^{-1}(k b)^{-1} X_{0} \\
& \left(1-\alpha^{2}\right)\left[-1-L n \varepsilon+A_{1}(\alpha)\right], \\
X_{0}= & {\left[-\left(1-\alpha^{2}\right)-\left(2-\alpha^{2}\right) L n \varepsilon\right.} \\
& \left.+\alpha^{2} A_{1}(\alpha)+2\left(1-\alpha^{2}\right) A_{2}(\alpha)\right]^{-1}, \\
\psi= & \Psi-\frac{3}{2} X_{0} \alpha^{2}\left(1-\alpha^{2}\right)\left[-1-L n \varepsilon+A_{1}(\alpha)\right]^{2}, \\
\Psi= & \frac{3}{2} Q^{-1}\left[2-\alpha^{2}-3 \alpha^{-1}+2 \alpha^{-1} L n(2 n \pi)\right. \\
& \left.-\left(1+\alpha^{2}\right) L n \varepsilon-2 A_{3}(\alpha)-\left(1-\alpha^{2}\right) A_{1}(\alpha)\right], \\
Q=1 & +\frac{\operatorname{Ln}\left(\frac{n \lambda}{d_{\text {head }}}\right)-\frac{3}{2}}{2 L n(2 \pi n)+2 L n\left(\frac{2 \pi \xi}{\lambda}\right)-4} \\
& +\alpha\left(1-\alpha^{2}\right) A_{1}(\alpha)+1-\alpha,
\end{aligned}
$$

$$
2 L n(2 n \zeta / \lambda)=-\alpha+\alpha^{3}+\alpha\left(1+\alpha^{2}\right) L n \varepsilon+2 \alpha A_{3}(\alpha) .
$$

According to Eq. (1), $\alpha$ (the ratio of helical tail's length to actual length of the helical tail) is one of the important parameters that influences the swimmer's forward velocity, $\varepsilon=\sqrt{e} \pi b \alpha / \lambda$ and $A_{[1,2,3]}$. Periodic integrals of the flow fields are signified by local Stokeslet functions. By varying the length of the helix, the actual length of the helical tail and the number of waves remain constant; however, the wavelength and amplitude of the helical tail change. Therefore, the variable parameters can be expressed as functions of fixed parameters. By using helical length coefficient $(\alpha)$, the wavelength and amplitude can be defined as follows:

$$
\begin{aligned}
& \alpha=\frac{L_{\text {tail }}}{L_{\text {actual }}}, \quad L_{\text {actual }}=\text { cons }=c \rightarrow L_{\text {tail }}=c . \alpha, \\
& L_{\text {tail }}=n . \lambda, \quad n=\mathrm{cons}=e \rightarrow L_{\text {tail }}=e \cdot \lambda
\end{aligned}
$$

where $L_{\text {tail }}$ is the helical tail's length, $L_{\text {actual }}$ is the actual length of the helical tail, and $c$ and $e$ are the constant parameters. By using Eqs. (7) and (8), the wavelength of the helical tail can be expressed as in Eq. (9):

$$
\lambda=\frac{c}{e} \cdot \alpha .
$$

On the other hand, according to [15], the helical length coefficient $(\alpha)$ is proportional to the amplitude and number of wavelengths of the helical tail, defined as follows:

$$
\begin{aligned}
& \alpha=\frac{1}{\sqrt{1+\left(k h_{\text {helix }}\right)^{2}}} \rightarrow h_{\text {helix }}=\left(\frac{1}{k}\right) \cdot\left[\frac{1}{\alpha^{2}}-1\right]^{1 / 2}, \\
& k=\frac{2 \pi}{\lambda} \rightarrow k=\frac{2 \pi e}{c . \alpha} \rightarrow h_{\text {helix }}=\frac{c \cdot \alpha}{2 \pi e}\left[\frac{1}{\alpha^{2}}-1\right]^{1 / 2}
\end{aligned}
$$

As is known, the value of coefficient $\alpha$ is always smaller than one. Thus, it is concluded from Eq. (10) that, with an increase in the helical tail's length, the amplitude of the helical tail $\left(h_{\text {helix }}\right)$ varies less than the wavelength and is small enough to be ignored. Therefore, based on Eqs. (1) to (10), it is concluded that the swimmer's forward velocity increases by increasing the helical tail length.

\section{Experimental verification}

\subsection{Laboratory prototype of the biomimetic swimmer robot}

In order to investigate the effect of the helical tail length on the forward velocity of swimmer and verification of theoretical results, an experimental prototype 


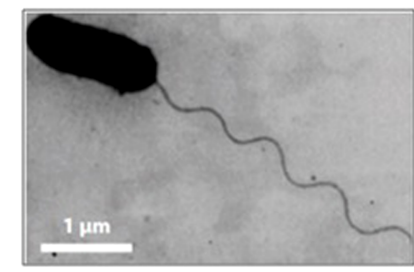

(a)

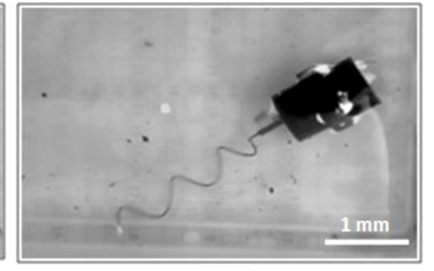

(b)
Figure 2. (a) The monotrichous bacteria with prokaryote propulsion [19]. (b) The fabricated biomimetic helical swimmer robot.

of a macro-size swimmer robot inspired by monotrichous bacteria [19] is fabricated. This swimmer robot consists of two main sections (body and tail), which are connected via a coupling. A view of this swimmer robot can be observed in Figure 2.

The swimmer body consists of two half bodies. There is enough space in each of these two casings for installing the needed equipment such as battery, motor, and connecting cables; these equipment can also be easily taken out when necessary. The propulsion system that drives this swimmer robot is a helical tail which, by spinning in a counterclockwise direction along the body's major axis, thrusts force to enable the swimmer to move forward. This propulsion system is connected to the swimmer body through a coupling, which keeps the helical tail in line with the body's central axis and transfers the motor's rotational velocity to the helix. Considering the scaled-up size of the swimmer prototype and in order to preserve the less-than-one Reynolds conditions, the PI Buckingham theorem was employed. The fluid used in this experiment was silicone oil, with a kinematic viscosity of $1000 \mathrm{cSt}$ and density of $971 \mathrm{~kg} / \mathrm{m}^{3}$. The body and helical tail of the considered swimmer robot were designed and fabricated using the smallest available equipment in the market, with the highest power transmission capability. The geometrical specifications of the swimmer and test equipment are listed in Table 1 . The test was performed in a rectangular tank made of Plexiglas with dimensions of $30 \times 30 \times 70 \mathrm{~cm}^{3}$.

\subsection{The influence of the helical length on forward velocity in a single helical swimmer robot}

In this paper, to emulate an upper-scale environment of micro-scale robots swimming at low Reynolds numbers, the liquid with high viscosity is used. The Reynolds number [20] can be expressed as a function of the relative object velocity to fluid, $U$, characteristic linear dimension, $L_{\text {total }}$, (defined herein as the overall length of swimmer robot (body length + helical tail)), fluid density, $\rho$, and fluid dynamic viscosity, $\mu$, as follows:

$$
\operatorname{Re}=\frac{\rho U L_{\mathrm{total}}}{\mu}
$$

Table 1. Specification of the helical swimmer and experimental setup.

\begin{tabular}{lcc}
\hline \multicolumn{1}{c}{ Sy mbol } & Value & Unit \\
\hline$d_{\text {head }}$ & 28 & $\mathrm{~mm}$ \\
$L_{\text {head }}$ & 42 & $\mathrm{~mm}$ \\
$2 b$ & 1 & $\mathrm{~mm}$ \\
$2 h_{\text {helix }}$ & 11 & $\mathrm{~mm}$ \\
$L_{\text {tail }}$ & 60 & $\mathrm{~mm}$ \\
$\lambda$ & 17 & $\mathrm{~mm}$ \\
$\beta$ & 63.7 & $\mathrm{deg}$ \\
Material of helix tail & Linear elastic & - \\
$W$ & 28 & $\mathrm{gr}$ \\
$D_{\text {motor }}$ & 6 & $\mathrm{~mm}$ \\
$L_{\text {motor }}$ & 18 & $\mathrm{~mm}$ \\
$V_{\text {motor }}$ & 3 & $\mathrm{~V}$ \\
vol & & $\mathrm{mm}$ \\
$V_{\text {battery }}$ & $3.6^{*} 18^{*} 24$ & $\mathrm{~m}$ \\
$\rho$ & 3.7 & $\mathrm{~kg} / \mathrm{m}^{3}$ \\
$\nu$ & 971 & $\mathrm{cSt}$ \\
\hline
\end{tabular}

In order to preserve the low Reynolds conditions, the specific helical tail lengths were selected. In this test, five different tail lengths at three different spinning frequencies were analyzed. In each test, the helical tail's length varies between $L_{1}$ to $L_{5}$ which, by varying the helical tail in this range, the wavelength and amplitude of the helix also vary. Then, for all helical tail's lengths, the forward velocity was measured. In order to estimate the forward velocity of the swimmer for these helical tails, the length of the channel was graduated; besides, their velocity was determined by using two Cannon cameras with the ability of capturing 100 frames per second (which were looking from the top and front views). A view of the swimmer's free motion in silicon oil can be seen in Figure 3. The distances between swimmer and container walls at both ends are large enough to ignore the wall effects. The test results for different tail lengths are presented in Table 2 .

The experimental results show that, by increasing

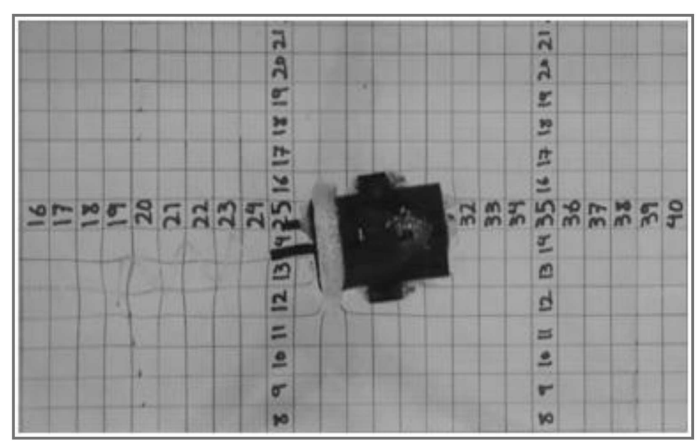

Figure 3. Photography of the helical swimmer in a rectangular container while swimming freely. 
Table 2. Experimental measurements.

\begin{tabular}{|c|c|c|c|c|c|c|c|}
\hline \multirow[t]{2}{*}{ Frequency } & \multicolumn{2}{|c|}{$\begin{array}{c}\text { The Length of } \\
\text { the helical tail } \\
(\mathrm{mm})\end{array}$} & \multicolumn{2}{|c|}{$\begin{array}{l}\text { The wave length } \\
\text { of the helical tail } \\
(\mathrm{mm})\end{array}$} & \multicolumn{2}{|c|}{$\begin{array}{c}\text { The amplitude of } \\
\text { the helical tail } \\
(\mathrm{mm})\end{array}$} & \multirow{2}{*}{$\begin{array}{c}\text { Forward } \\
\text { velocity value } \\
(\mathbf{m m} / \mathbf{s})\end{array}$} \\
\hline & $L_{1}$ & 46 & $\lambda_{1}$ & 17 & $h_{1}$ & 6 & \\
\hline \multirow{4}{*}{$F=2 \mathrm{~Hz}$} & $L_{2}$ & 55 & $\lambda_{2}$ & 20 & $h_{2}$ & 5.8 & 3.55 \\
\hline & $L_{3}$ & 60 & $\lambda_{3}$ & 23 & $h_{3}$ & 5.5 & 4.8 \\
\hline & $L_{4}$ & 70 & $\lambda_{4}$ & 25 & $h_{4}$ & 5.2 & 6.5 \\
\hline & $L_{5}$ & 78 & $\lambda_{5}$ & 27 & $h_{5}$ & 5 & 8.35 \\
\hline \multirow{5}{*}{$F=3 \mathrm{~Hz}$} & $L_{1}$ & 46 & $\lambda_{1}$ & 17 & $h_{1}$ & 6 & 5.125 \\
\hline & $L_{2}$ & 55 & $\lambda_{2}$ & 20 & $h_{2}$ & 5.8 & 6.8 \\
\hline & $L_{3}$ & 60 & $\lambda_{3}$ & 23 & $h_{3}$ & 5.5 & 7.35 \\
\hline & $L_{4}$ & 70 & $\lambda_{4}$ & 25 & $h_{4}$ & 5.2 & 9.75 \\
\hline & $L_{5}$ & 78 & $\lambda_{5}$ & 27 & $h_{5}$ & 5 & 12.4 \\
\hline \multirow{5}{*}{$F=4 \mathrm{~Hz}$} & $L_{1}$ & 46 & $\lambda_{1}$ & 17 & $h_{1}$ & 6 & 7.2 \\
\hline & $L_{2}$ & 55 & $\lambda_{2}$ & 20 & $h_{2}$ & 5.8 & 8.25 \\
\hline & $L_{3}$ & 60 & $\lambda_{3}$ & 23 & $h_{3}$ & 5.5 & 10.15 \\
\hline & $L_{4}$ & 70 & $\lambda_{4}$ & 25 & $h_{4}$ & 5.2 & 12.65 \\
\hline & $L_{5}$ & 78 & $\lambda_{5}$ & 27 & $h_{5}$ & 5 & 16.5 \\
\hline
\end{tabular}

the helical tail's length, the wavelength $(\lambda)$ and the amplitude of the helical tail ( $\left.h_{\text {helix }}\right)$ change; however, the amplitude of the helical tail varies less than the wavelength and can be ignored in the calculations. In order to verify the agreement between theoretical and experimental tests, the forward velocities of the swimmer were calculated by using Eq. (1) for five different helical lengths at three different spinning frequencies. According to Figure 4, the theoretical results agree with the experimental data, and the obtained results indicate that the swimmer's forward velocity increases by increasing the helical tail's length.

Figure 4 shows that the predicted theoretical values are larger than the experimental results. The main assumptions made in this article, which illustrate the deviation between the theory and experiment results, are as follows. It was assumed herein that the swimmer head is elliptical, which is probably more important. Another assumption was defined in the theoretical calculation in which the walls are far enough so that they will not significantly affect the behavior of swimmer motion. Herein, all interactions between the swimmer's body and helical tail have been ignored. Another factor contributing to the difference between the theory and experiment is that the uncertainty in the experimental data is due to the uncertainties associated with the measurement equipment and the user.

\section{Introducing the Double Helices Propulsion Mechanism (DHPM)}

Considering the validated results of the preceding section, a system is introduced herein, which can produce various forward velocities during swimming by altering the helical tail's length. This mechanism (called DHPM) consists of two parallel helices. The outer helix, introduced in the previous section, rotates in the same direction at similar frequencies. A schematic of this swimmer, which has the mentioned helical propulsion mechanism and includes a cylindrical head and a double helices system, is depicted in Figure 5. This performance (change of tail length) can also be seen in prokaryotic microorganisms such as $E$. coli in their "run and tumble" behavior during locomotion. During the run motion, the flagellum spins in the counterclockwise direction and the microorganism moves along a straight path. During the tumble, the flagellum, which rotates in the clockwise direction, becomes semi-coiled, thus reducing the velocity of the microorganism $[6,21]$.

\subsection{Designing the Shape Memory Alloy (SMA) helix}

As smart materials, Shape Memory Alloys (SMA) are able to return to their original shape when heated. Due to their large deformation, low actuation voltage, high biocompatibility, and noiseless and uniform actuation, 


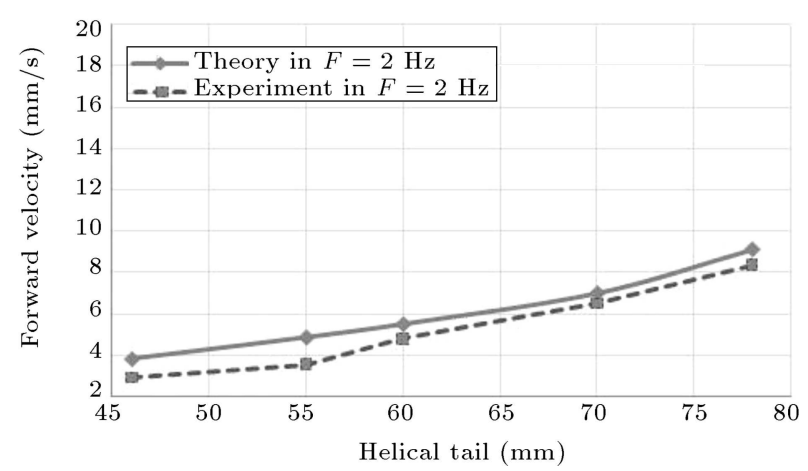

Table 3. The material properties of Shape Memory Alloy (SMA) spring [28].

\begin{tabular}{lcc}
\hline Parameters & Value & Unit \\
\hline$\rho$ & 6.45 & $\frac{\mathrm{gr}}{\mathrm{cm}^{3}}$ \\
$G_{M}, G_{A}$ & 28,83 & $\mathrm{GPa}$ \\
$A_{s}, A_{f}$ & 70,90 & ${ }^{\circ} \mathrm{C}$ \\
$M_{s}, M_{f}$ & 65,45 & ${ }^{\circ} \mathrm{C}$ \\
$\epsilon_{L}$ & $6 \%$ & - \\
$\tau_{A}, \tau_{M}$ & 120,75 & $\mathrm{MPa}$ \\
$C_{p}$ & 0.2 & $\frac{\mathrm{cal}}{\mathrm{gr} .}{ }^{\circ} \mathrm{C}$ \\
$\rho_{M}, \rho_{A}$ & 100,80 & $\Omega . \mathrm{cm}$ \\
\hline
\end{tabular}
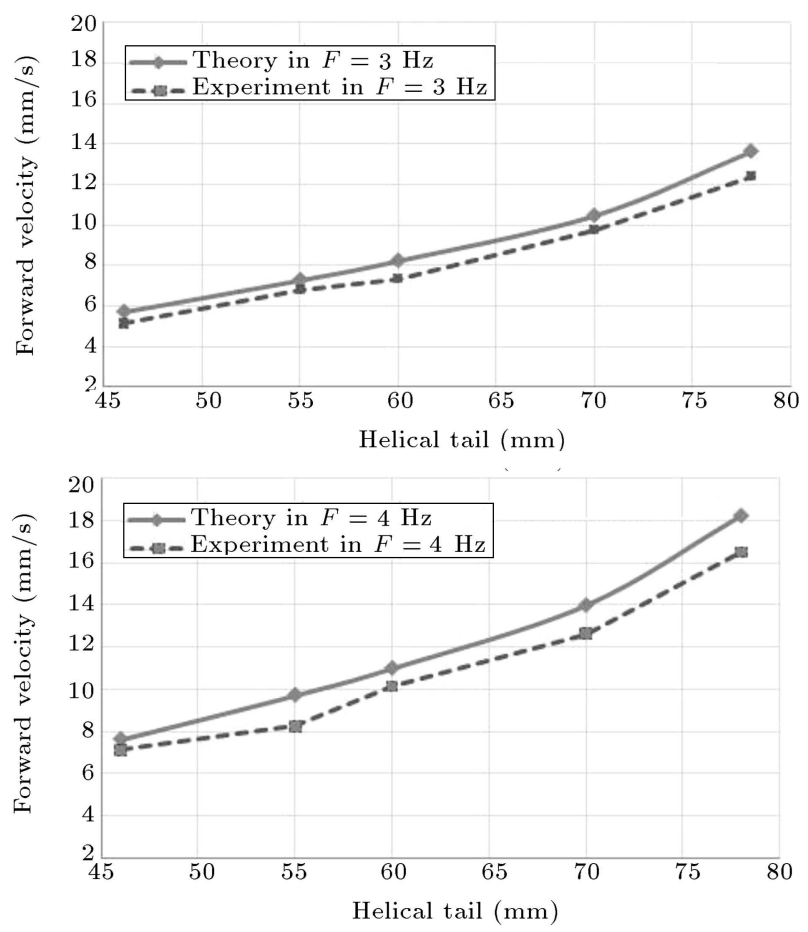

Figure 4. Comparison between experimental and theoretical forward velocities for different helical lengths in different spinal propulsive frequencies.

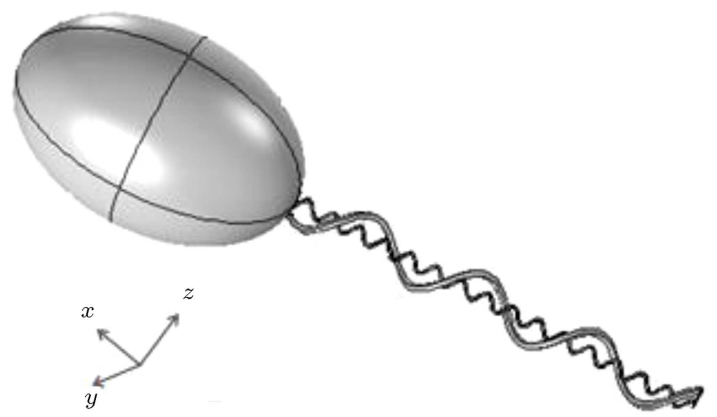

Figure 5. Illustration of the helical propulsion mechanism in helical swimmer.

the use of these materials in actuators has become fascinating [22-28]. Due to larger displacement in shape memory springs, they have been selected to be used in the helical propulsion system rather than shape memory wires. A type of Ti-Ni SMA has been

selected for the helical tail of this swimmer, which is an important type of SMA with many advantages including noiseless actuation, large deformation, large recovery strain $(<6 \%)$, high biocompatibility, and low actuation voltage [28]. Table 3 indicates the main properties of the SMA spring [29].

In order to obtain an extreme forward velocity, the minimum and maximum values of desired helical length are chosen based on the verified results in Section 3. Therefore, the range of the desired length is considered to be between 46 to $60 \mathrm{~mm}$ at spinal propulsive frequency of $3 \mathrm{~Hz}$. A schematic of length variations in the helical propulsion system is shown in Figure 6.

As shown in Figure 7, there are two cases for the above propulsion system:

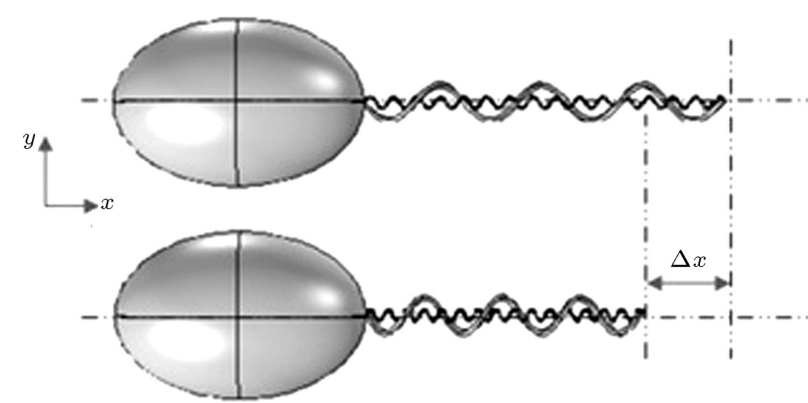

Figure 6. The schematic of the varying length in the helical propulsion system by using SMA helix.

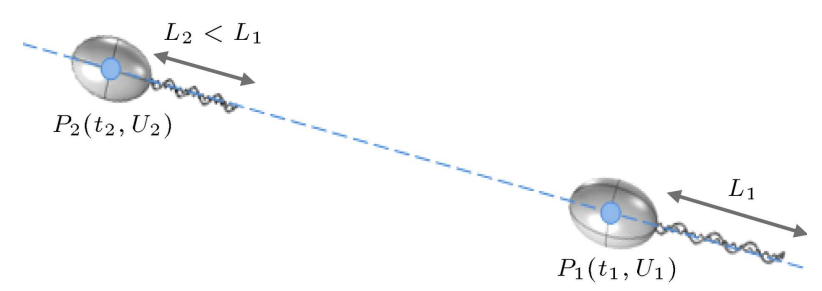

Figure 7. Two modes for the Double Helices Propulsion Mechanism (DHPM). In $P_{1}$ position, the helical swimmer robot has the maximum length and can move by maximum forward velocity, while, in $P_{2}$ position, the helical swimmer robot has the minimum length and can move by a minimum forward velocity. 
1. Case of maximum length;

2. Case of minimum length.

In the first case, the outer helix, which acts as a passive helix, has its usual primary length, and the inner helix is at its secondary length. In the second case, following the application of heat to the inner helix, this helix is compressed which leads to the passive helix compression; next, the whole propulsion system reaches to its minimum mode. By using the energy stored in this helix, the length of the whole propulsion system can reach the maximum length (initial length).

Through Eqs. (12)-(16) [30], the SMA helix can be designed. In Eq. (10), $F_{P}$ is the force generated by outer helix (propulsion helical tail), which is applied to the SMA helix, $2 h_{\mathrm{SMA}}$ helix is the average diameter of the spring, and $2 b_{\mathrm{SMA}}$ represents the SMA wire diameter; in Eq. (12), $C$ is the spring index defined as follows:

$$
\begin{aligned}
& b_{\mathrm{SMA}}=\left(\frac{2 F_{P} W C}{\pi \tau_{c}}\right)^{1 / 2}, \\
& C=\frac{\text { The average diameter of the spring }}{\text { The SMA wire diameter }}=\frac{2 h_{\mathrm{SMA}}}{2 b_{\mathrm{SMA}}} .
\end{aligned}
$$

$W$ is known as the Wahl correction factor, which has a value of 1.40375 for a spring index of 4 and $\tau_{c}$ is the maximum stress applied to the helix:

$$
W=\frac{4 C-1}{4 C-4}+\frac{0.615}{C} .
$$

Furthermore, the number of turns in SMA helix can be obtained from the following equation as follows:

$$
\begin{aligned}
& n_{\mathrm{SMA}}=\frac{b_{\mathrm{SMA}} \cdot \Delta x}{4 \pi\left(h_{\mathrm{helix}}\right)^{2} \cdot \Delta \gamma}, \\
& \Delta \gamma=\gamma_{H}-\gamma_{L} .
\end{aligned}
$$

In the above equation, $\Delta x$ denotes the displacement between the minimum and maximum ranges of the desired length, and $\Delta \gamma$ is the difference between strains at low and high temperatures. Figure 8 shows the geometrical parameters of the designing Double Helices Propulsion Mechanism (DHPM).

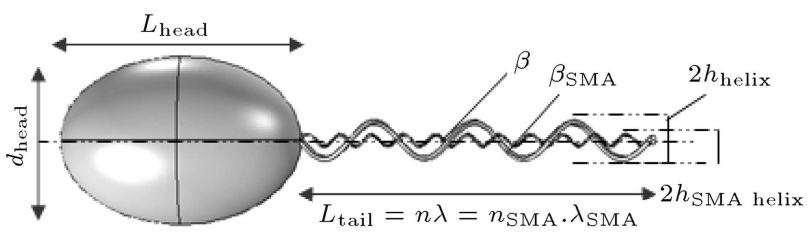

Figure 8. The geometrical parameters of the Double Gelices Propulsion Mechanism (DHPM).

\subsection{Actuation of the SMA helix for generating variable velocities}

There are different techniques for the actuation of shape memory alloys. In this investigation, a temperature variation is used to induce the phase changes in the SMA helix. For this purpose, the inner SMA helix is heated by supplying electricity to a resistive wire attached to it. According to Section 2, the variation of helical wave amplitude ( $h_{\text {helix }}$ ), due to the change of helical length, is small enough to ignore. Therefore, to simplify the thermal equations, it can be assumed that the temperature has been distributed only along the helical length direction. When minimum tail length is required, the SMA helical tail is electrically heated to raise its temperature from $T_{0}$ to $T_{a f}$ (Austenit finish temperature), in which case the length of the inner helix is shortened and, along with it, the outer helix is compressed as well. Thus, the entire length of the helical propulsion system is compressed from $L_{2}$ to $L_{1}$, in which the swimmer has the minimum forward velocity. To calculate the desired voltage in order to reach $T_{a f}$ (Austenit finish temperature), the heat transfer rate in heating the SMA helical tail can be expressed as in the following equation:

$$
\begin{aligned}
\int \frac{U(t)^{2}}{R} d t= & \int h_{\text {spring }} A\left[T(t)-T_{0}\right] d t \\
& +\int c \rho\left(\frac{\pi d^{2}}{4}\right) l d T(t),
\end{aligned}
$$

where $U(t)$ is the voltage supplied to SMA helical tail $(\mathrm{V}), R$ is the electrical resistance, $C_{p}$ is the specific heat coefficient, and $T(t)$ (in ${ }^{\circ} \mathrm{K}$ or ${ }^{\circ} \mathrm{C}$ ), $\rho$, and $h_{\text {sprimg }}$ are the temperature, density, and the heat transfer coefficient of the of SMA helical tail, respectively. Figure 9 shows the voltage required to reach the austenite temperature. The result shows that in order to reach minimum helical length in the austenite temperature, 10.72 volt should be supplied to SMA helix. According to the defined mission, to obtain a constant forward velocity, the total length of

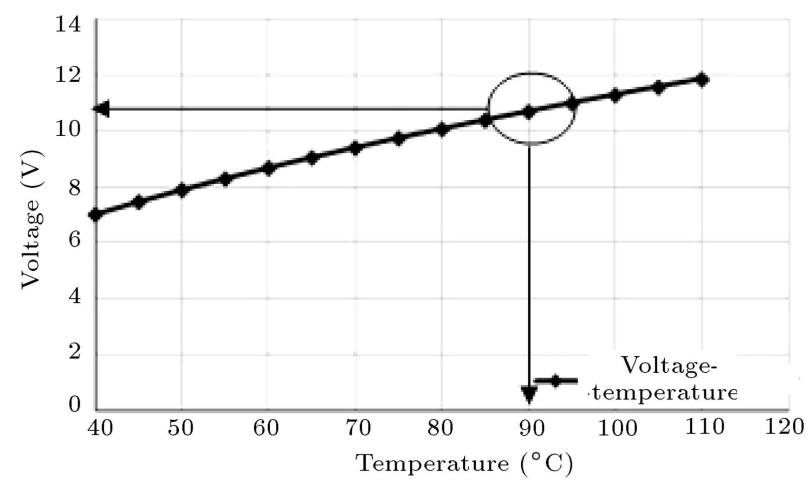

Figure 9. The required voltage to actuate SMA helical tail to obtain austenite temperature. 


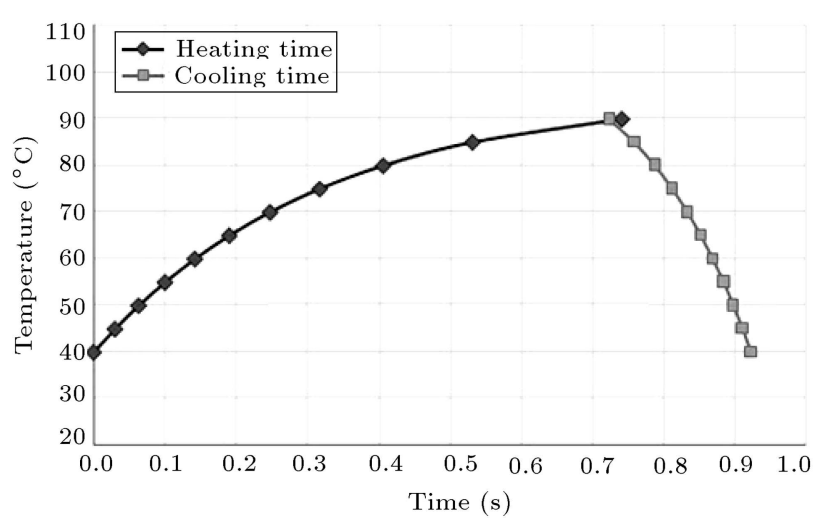

Figure 10. Simulation curves of the SMA helical tail used in here.

the helical propulsion systems can be maintained at the desired length by controlling the input current and keeping the final temperature constant. In order to reach the maximum velocity, the current is cut off and the system is cooled to a temperature of $T_{m s}$ (martensit start temperature). Thus, by cooling the SMA helix, the stored energy in the outer helix (propulsion helical tail) is released, and the entire length of the propulsion system is increased to its maximum value $(60 \mathrm{~mm})$. In the cooling period of the SMA helix, the following equation is used to describe the heat transfer:

$$
\int h_{\text {spring }} A\left[T(t)-T_{0}\right] d t+\int c \rho\left(\frac{\pi d^{2}}{4}\right) l d T(t)=0 .
$$

By using Eqs. (17) and (18), the cooling and heating times can be estimated. Figure 10 shows the cooling and heating periods of the SMA helix in one cycle.

According to the simulation curve, in order to reach minimum length or return to the primary position in maximum length, a short time delay in heating time or cooling time should occur in the swimmer robot motion. Through repeating this process, the helical swimmer can reach variable velocities at different times.

\section{Performance analyses}

With regard to the specifications of the designed helices, since the ratio of the outer diameter of the SMA helix corresponds to propulsion helix $\left(\frac{D_{\text {SMA helix }}}{D_{\text {helix }}}=\right.$ 0.17 ), the hydrodynamic interaction between two helices can be ignored [16,31], and it can be assumed that the two helices perform independently. As was mentioned in the previous section, the above swimmer consists of a cylindrical body with diameter $d_{\text {head }}$ and length $L_{\text {head }}$, connected to the helices via a coupling (Figure 8). The outer helical tail acts as the propulsion system, and the inner helix (made of SMA material) controls the forward velocity of the swimmer. Both helices have the same rotational axes and spin in the
Table 4. The specification of the designed helices in the constant spinal propulsive frequency (3 HZ).

\begin{tabular}{lcc}
\hline \multicolumn{1}{c}{ Parameters } & Value & Unit \\
\hline$d_{\text {head }}$ & 28 & $\mathrm{~mm}$ \\
$L_{\text {head }}$ & 42 & $\mathrm{~mm}$ \\
$L_{\text {tail }}$ & $46-60$ & $\mathrm{~mm}$ \\
$n, n_{\text {SMA }}$ & $3.53,15.4$ & - \\
$2 b, 2 b_{\text {SMA }}$ & $1,0.46$ & $\mathrm{~mm}$ \\
$2 h_{\text {helix }}, 2 h_{\text {SMA helix }}$ & $11,1.871$ & $\mathrm{~mm}$ \\
$\lambda, \lambda_{\text {SMA }}$ & Variable & $\mathrm{mm}$ \\
$\beta, \beta_{\text {SMA }}$ & Variable & $\mathrm{deg}$ \\
\hline
\end{tabular}

same direction at similar frequencies. The geometrical specifications of the designed helices are given in Table 4. In order to analyze the swimmer's performance following adding the Double Helices Propulsion Mechanism (DHPM), the motion equations of swimmer can be used.

For the system studied in Section 2 and by considering insignificant inertial forces at this scale, the equations for conservation of linear and angular momenta are as follows:

$$
\begin{aligned}
& \int_{0}^{n \lambda} d F_{x}^{\mathrm{vis}}+F_{x}^{\mathrm{head}}=F_{x}^{e} \\
& \int_{0}^{n \lambda} d M_{x}^{\mathrm{vis}}+M_{x}^{\mathrm{head}}=M_{x}^{e}
\end{aligned}
$$

In the above equation, $d F_{x}^{\mathrm{vis}}$ is the propulsive force in $x$ direction, and $d M_{x}^{\mathrm{vis}}$ is the torque resulting from fluid reaction on the helical tail. $F_{x}^{\text {head }}$ and $M_{\text {head }}^{i}$, respectively, denote the viscous drag and torque acting on the head. $F_{x}^{e}$ and $M_{x}^{e}$ are the external forces and torques that affect the swimmer in different directions which are equal to zero herein due to the assumption of a self-propelled swimmer robot. According to the resistive force theory $[3,7,11,32]$, it can be assumed that the normal and tangential components of hydrodynamic forces act on a cylindrical element of length $d s$, given by [11]:

$$
\left[\begin{array}{l}
d f_{n} \\
d f_{t}
\end{array}\right]=\left[\begin{array}{cc}
-\xi_{n} & 0 \\
0 & -\xi_{t}
\end{array}\right] \cdot\left[\begin{array}{l}
v_{n} \\
v_{t}
\end{array}\right] d s
$$

where $\xi_{n}$ and $\xi_{t}$ are the corresponding coefficients of resistance:

$$
\xi_{t}=\frac{2 \pi \mu}{\ln \left(\frac{n \lambda}{b}\right)-1 / 2}, \quad \xi_{n}=\frac{4 \pi \mu}{\ln \left(\frac{n \lambda}{b}\right)+1 / 2} .
$$

In the above equation, $n \lambda$ and $b$ denote the helical tail's 
length and the helix radius, respectively. The local velocity components affecting a helix element in the normal and tangential directions are represented as $v_{n}$ and $v_{t}$; then, we have:

$$
\begin{aligned}
& {\left[\begin{array}{l}
v_{n} \\
v_{t}
\end{array}\right]=\left[\begin{array}{cc}
\cos (\beta) & -\sin (\beta) \\
\sin (\beta) & \cos (\beta)
\end{array}\right] \cdot\left[\begin{array}{c}
A(\omega-\Omega) \\
U
\end{array}\right],} \\
& {\left[\begin{array}{l}
d f_{x}^{\text {vis }} \\
d f_{y}^{\text {vis }}
\end{array}\right]=\left[\begin{array}{cc}
\cos (\beta) & -\sin (\beta) \\
\sin (\beta) & \cos (\beta)
\end{array}\right] \cdot\left[\begin{array}{l}
d f_{n} \\
d f_{t}
\end{array}\right] .}
\end{aligned}
$$

If the number of the helical waves formed by the tail is $n$, then by integration with Eq. (24), the thrust force and the required torque of helix tail can be written as follows:

$$
F_{x}^{\mathrm{vis}}=\int_{0}^{n \lambda} d f_{x}^{\mathrm{vis}}, \quad M_{x}^{\mathrm{vis}}=\int_{0}^{n \lambda} d M_{x}^{\mathrm{vis}} .
$$

There would be an additional viscous torque acting on element $d s$ of the tail that results from the fluid reaction with the element of the tail. $x$ component of this torque is [32]:

$$
d M_{\text {tail }}=4 \pi \mu h_{\text {helix }}^{2} \Omega \cos \beta d x .
$$

By using Eqs. (25), (26), a linear relationship relating the total hydrodynamic force and torque to rigid body velocity $(U)$ and angular velocity $(\Omega)$ of the swimmer can be determined for each helix:

$$
\left[\begin{array}{ll}
P_{11} & P_{12} \\
P_{21} & P_{22}
\end{array}\right]\left[\begin{array}{l}
U \\
\Omega
\end{array}\right]=\left[\begin{array}{l}
F \\
M
\end{array}\right]
$$

In Appendix A, the solution for forward velocity $(U)$ and angular velocity $(\Omega)$ can be found by substituting the values of the hydrodynamic matrixes of body and helix. By using the calculated thrust force and the required torque of helix tail in Eq. (25), the power efficiency of helical swimmer robot can be defined as follows $[3,7,32]$ :

$$
\eta=\frac{F \cdot U}{M \cdot \omega}
$$

In the diagram of Figure 11, the forward velocity and power efficiency of the helical swimmer with a double helices propulsion system have been plotted versus various tail lengths.

Figure 11(a) indicates that the forward velocity of swimmer for single- and double-helix propulsion systems increases by varying the helical tail length in the considered range. Moreover, through the comparison of the above diagram with the graph, which displays the forward velocity of swimmer with a single helix, it is observed that the increase in the number of helices leads to an increase in the swimmer's forward velocity. The variation of the power efficiency with a helical tail
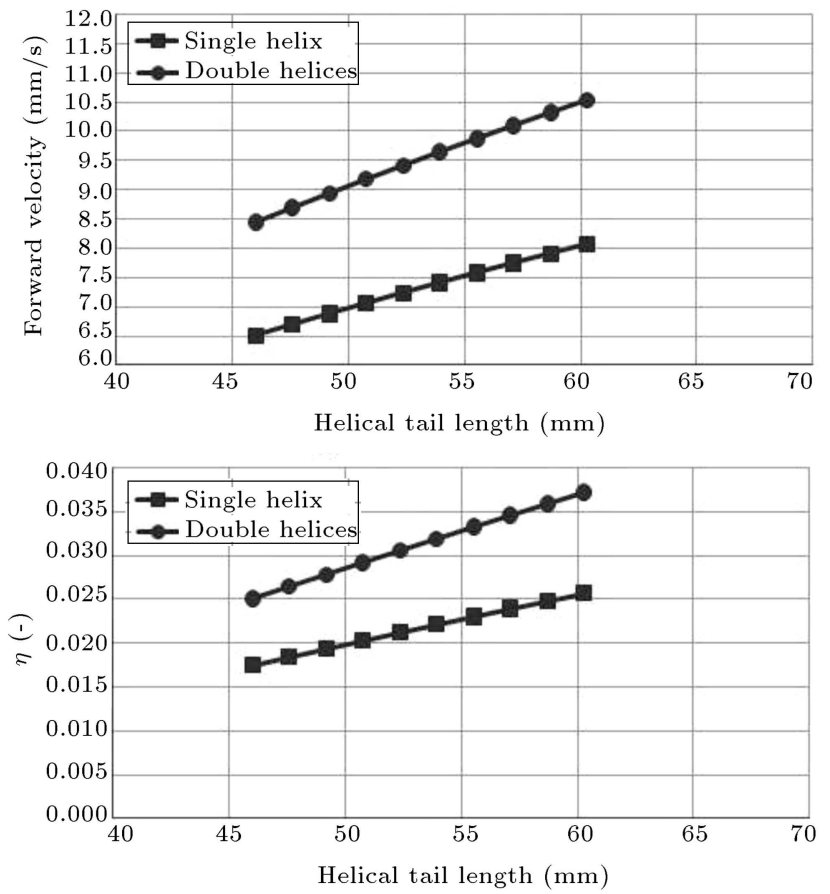

Figure 11. Simulation curves of the performance of the double helices propulsion mechanism by RFT method in swimmer robot: (a) Forward velocity and (b) power efficiency.

length for the single- and double-helix propulsion systems is shown in Figure 11(b). As shown in this figure, in the single helix, the efficiency is about $\eta \cong 1.7-2.5 \%$, showing that the efficiency in this case is completely dependent on the helical tail length, and this value is found to be $2 \%$ for similar propulsive microorganism (Escherichia coli) in $[3,7]$. The application of the Double Helices Propulsion System (DHPS) leads to an increase in the efficiency such that, for the singlehelix propulsion system, maximum swimming efficiency is $2.5 \%$, whereas it is $3.7 \%$ for the double helices propulsion system in the maximum length. According to the obtained results, by applying the double helices mechanism as a propulsion system, it is observed that the designed helical swimmer robot has the ability to change the forward velocity during swimming, whose performance somehow increased; moreover, it can be applied to different variable velocities.

\section{Conclusion}

Many researchers have already demonstrated that helical propulsion is an appropriate and practical method for swimming in the low Reynolds conditions. In addition to frequency, the other geometric parameters can be effective in the forward velocity of helical swimmer. According to the theoretical and experimental researches, the most important geometrical parameter with a significant effect on swimmer velocity is the length of helical tail. By using this characteristic, the 
hydrodynamic model based on Slender Body Theory (SBT) is implemented, and attempt is made to clarify that, by varying the helical length, the wavelength and amplitude of the helical tail change simultaneously; the actual length of the helical tail and the number of waves remain constant.

It was concluded that, with an increase in the helical tail's length, the amplitude of the helical tail varies less than the wavelength and is small enough to ignore; moreover, by fabricating a scaled-up macrodimension prototype with a single helical tail and Reynolds number of less than one, our results were verified. By means of these results, a new mechanism made of SMA material was designed and proposed. The proposed mechanism can change the forward velocity of the swimmer by varying the geometrical parameters of its helical tail where the swimmer can achieve the variable forward velocities that are required by its predefined missions. Then, the performance of the double helices system, such as forward velocity and efficiency, was estimated by dynamic modelling of the motion in different tail lengths; moreover, the performances of the single- and double-helix propulsion systems were compared. The results indicate that this mechanism increases the forward velocity and efficiency and produces variable forward velocities at each frequency. Therefore, the tail length variation method can be used by itself to change the swimmer velocity; besides, it can also be employed as an auxiliary method along with frequency variation to increase the velocity range of swimmer robots with helical tails. According to the obtained results, through applying a double helices mechanism as propulsion system, it was concluded that the designed helical swimmer robot has the ability to change the forward velocity of a swimmer robot during swimming, whose performance somehow increases and can be applied in different complex conditions. In future works, fluidic coupling effects among double helices in different helical diameters and various spacing between helices will be investigated. Moreover, by manufacturing a swimmer with this propulsion system, we will investigate the controlling of forward velocity in this type of swimmers for the implementation of predefined missions and try to solve the designing challenge.

\section{Nomenclature}

$\begin{array}{ll}d_{\text {head }} & \text { Cylindrical head diameter }(\mathrm{mm}) \\ L_{\text {head }} & \text { Cylindrical head length }(\mathrm{mm}) \\ 2 b, 2 b_{\text {SMA }} & \begin{array}{l}\text { Helical tail diameter and SMA helical } \\ \text { tail diameter }(\mathrm{mm})\end{array} \\ 2 h_{\text {helix }} & \text { Helical wave amplitude } \\ 2 h_{\text {SMA helix }} & \text { SMA Helical wave amplitude }(\mathrm{mm})\end{array}$

\begin{tabular}{|c|c|}
\hline$L_{\text {tail }}$ & helical tail's length (mm) \\
\hline$L_{\text {actual }}$ & Actual helical tail's length (mm) \\
\hline$L_{\text {total }}$ & $\begin{array}{l}\text { Overall length of swimmer robot (body } \\
\text { length }+ \text { helical tail) }(\mathrm{mm})\end{array}$ \\
\hline$\alpha$ & Helical length coefficient (deg) \\
\hline$\lambda, \lambda_{\mathrm{SMA}}$ & $\begin{array}{l}\text { Helical wave length and SMA Helical } \\
\text { wave length }(\mathrm{mm})\end{array}$ \\
\hline$n, n_{\mathrm{SMA}}$ & $\begin{array}{l}\text { Number of wavelengths and SMA } \\
\text { number of wavelengths (-) }\end{array}$ \\
\hline$W$ & Total weight $(\mathrm{gr})$ \\
\hline$D_{\text {motor }}$ & DC-motor diameter (mm) \\
\hline$L_{\text {motor }}$ & DC-motor length (mm) \\
\hline$V_{\text {motor }}$ & Voltage of motor $(\mathrm{V})$ \\
\hline vol battery $_{\text {b }}$ & Volume of battery $\left(\mathrm{m}^{3}\right)$ \\
\hline$V_{\text {battery }}$ & Voltage of battery (V) \\
\hline$\rho$ & Density of test fluid $\left(\mathrm{kg} / \mathrm{m}^{3}\right)$ \\
\hline$v$ & Kinematic viscosity $(\mathrm{cSt})$ \\
\hline$f$ & Spinal propulsive frequency $(\mathrm{Hz})$ \\
\hline$\psi, \Psi, Q$ & Velocity reduction function (-) \\
\hline$A_{[1,2,3]}$ & $\begin{array}{l}\text { Periodic integrals of the flow fields } \\
\text { signified by local Stokeslet functions } \\
(-)\end{array}$ \\
\hline$k$ & Wave number $\left(\mathrm{mm}^{-1}\right)$ \\
\hline$\rho_{\mathrm{SMA}}$ & Density of SMA helix $\left(\mathrm{kg} / \mathrm{m}^{3}\right)$ \\
\hline$G_{M}, G_{A}$ & $\begin{array}{l}\text { Modulus of elasticity martensite and } \\
\text { austenite }(\mathrm{GPa})\end{array}$ \\
\hline \multicolumn{2}{|c|}{$A_{S}, A_{f}, M_{S}, M_{f}$ Transformation temperatures $\left({ }^{\circ} \mathrm{C}\right)$} \\
\hline$\epsilon_{L}$ & Maximum transformation strain (-) \\
\hline$\tau_{M}, \tau_{A}$ & $\begin{array}{l}\text { Critical stress for martensite and } \\
\text { austenite }(\mathrm{MPa})\end{array}$ \\
\hline$C_{p}$ & Specific heat $\left(\mathrm{cal} /\left(\mathrm{gr} .{ }^{\circ} \mathrm{C}\right)\right)$ \\
\hline$\rho_{M}, \rho_{A}$ & $\begin{array}{l}\text { Electrical Resistivity for martensite } \\
\text { and Austenite }(\Omega . \mathrm{mm})\end{array}$ \\
\hline$h_{\text {sprimg }}$ & $\begin{array}{l}\text { Heat transfer coefficient of the of SMA } \\
\text { helical tail }\end{array}$ \\
\hline$C$ & Spring index (-) \\
\hline$F_{p}$ & $\begin{array}{l}\text { Force generated by outer helix } \\
\text { (propulsion helical tail) }(\mathrm{mmN})\end{array}$ \\
\hline$W$ & Wahl correction factor $(-)$ \\
\hline$\tau_{c}$ & $\begin{array}{l}\text { Maximum stress applied to the helix } \\
(\mathrm{MPa})\end{array}$ \\
\hline$\Delta x$ & $\begin{array}{l}\text { Difference between minimum and } \\
\text { maximum ranges of desired length } \\
(\mathrm{mm})\end{array}$ \\
\hline$\Delta \gamma$ & $\begin{array}{l}\text { Difference between strains at low and } \\
\text { high temperatures (-) }\end{array}$ \\
\hline$d F_{x}^{\mathrm{vis}}$ & Propulsive force in $x$ direction $(\mathrm{mN})$ \\
\hline
\end{tabular}




\begin{tabular}{|c|c|}
\hline$d M_{x}^{\text {vis }}$ & $\begin{array}{l}\text { Torque resulting from fluid reaction } \\
\text { with the helical tail }(\mathrm{mN} . \mathrm{mm})\end{array}$ \\
\hline$F_{x}^{\text {head }}, M_{x}^{\text {head }}$ & $\begin{array}{l}\text { Viscous drag and torque acting on the } \\
\text { head }(\mathrm{mN} / \mathrm{mN} . \mathrm{mm})\end{array}$ \\
\hline$F_{x}^{e}, M_{x}^{e}$ & $\begin{array}{l}\text { External forces and torques that affect } \\
\text { the swimmer }(\mathrm{mN} / \mathrm{mN} . \mathrm{mm})\end{array}$ \\
\hline$d f_{n}, d f_{t}$ & $\begin{array}{l}\text { Hydrodynamic forces acting on a } \\
\text { cylindrical element of local length } \\
(\mathrm{mN})\end{array}$ \\
\hline$\xi_{n}, \xi_{t}$ & $\begin{array}{l}\text { Local drag coefficient for motion } \\
\text { normal and tangential to local length } \\
(-)\end{array}$ \\
\hline$v_{n}, v_{t}$ & $\begin{array}{l}\text { Components of local normal and } \\
\text { tangential to local length }(\mathrm{mm} / \mathrm{s})\end{array}$ \\
\hline$U$ & $\begin{array}{l}\text { Velocity of the swimmer head, } \\
U=\left(U_{x}, U_{y}, U_{z}\right)(\mathrm{mm} / \mathrm{s})\end{array}$ \\
\hline$\Omega$ & $\begin{array}{l}\text { Rotation rate of the swimmer head, } \\
\Omega=\left(\Omega_{x}, \Omega_{y}, \Omega_{z}\right)(\mathrm{rad} / \mathrm{s})\end{array}$ \\
\hline
\end{tabular}

\section{References}

1. Abbot, J., Nagy, Z., Beyeler F., and Nelson, B. "Robotics in the small", IEEE Robotics \& Automation Magazine, 14(2), pp. 92-103 (June, 2007).

2. Feng, J. and Kwon Cho, S. "Mini and micro propulsion for medical swimmers", Micro Machines, 5, pp. 97-113 (2014).

3. Edd, J., Payen, S., Rubinsky, B., Stoller, M.L., and Sitti, M. "Biomimetic propulsion for a swimming surgical micro-robot", IEEE/RSJ Intelligent Robotics, 3, pp. 2583-2588 (2003).

4. Purcell, E.M. "Life at low Reynolds number", American J. Physics, 45(1), pp. 3-11 (1977).

5. Purcell, E.M. "The efficiency of propulsion by a rotating flagellum", The National Academy of Sciences of the USA (PNAS), 94(21), pp. 11307-11311 (1997).

6. Darnton, N.C., Turner, L., Rojevsky, S., and Berg, H.C. "On torque and tumbling in swimming Escherichia coli", Journal of Bacteriology, 189(5), pp. 1756-1764 (2007).

7. Chattopadhyay, S., Moldovan, R., Yeung, C., and Wu, X.L. "Swimming efficiency of bacterium Escherichia coli", PNAS, 103(37), pp. 13712-13717 (2006).

8. Behkam, B. and Sitti, M. "Design methodology for biomimetic propulsion of miniature swimming robot", Trans. ASME J. Dyn. Sys. Meas. Control, 128, pp. 36-43 (2006).

9. Xu, T., Hwang, G., Andreff, N., and Régnier, S. "Influence of geometry on swimming performance of helical swimmers using DoE", Springer, Journal of Micro-bio Robotics, 11(1-4), pp. 57-66 (2015).

10. Temel, F. and Yesilyurt, S. "Characterization and modeling of micro swimmers with helical tails and cylindrical heads inside circular channels", ASME Conference, 16-19 June (2013).
11. Gray, J. and Hancock, G.J. "The propulsion of seaurchin spermatozoa", J. Exp. Biol., 32, pp. 802-814 (1955).

12. Lighthill, J. "Flagellar hydrodynamics: the John von Neumann lecture", SIAM Rev., 18(2), pp. 161-230 (1976).

13. Taylor, G.I. "Analysis of the swimming of microscopic organisms", P. Roy. Soc. Lond. A Mat., 209(1099), pp. 447-461 (1951).

14. Batchelor, G.K. "Slender-body theory for particles of arbitrary cross-section in Stokes flow", Journal of Fluid Mechanics, 44(03), p. 419 (1970).

15. Brennen, C. and Winet, H. "Fluid mechanics of propulsion by cilia and flagella", Annul. Rev. Fluid Mech., 9, pp. 339-398 (1977).

16. Garica, J., Torre, D.L., and Bloomfield, V.A. "Hydrodynamic theory of swimming of flagellated microorganism", Biophysical Journal, 20, pp. 49-67 (1977).

17. Johnson, R. and Brokaw, C. "Flagellar hydrodynamics: A comparison between resistive-force theory and slender-body theory", Biophysical Journal, 25(1), pp. 113-127 (1979).

18. Fei, Y., Yu, H., and Burrows, B. "A review of methods for hydrodynamic analysis of helical swimming flagella", 18th International Conference on Automation and Computing (ICAC), pp. 1-8 (2012).

19. McCarter, L., Hilmen, M., and Silverman, M. "Flagellar dynamometer controls swarmer cell differentiation of V. parahaemolyticus", Cell, 54, pp. 345-351 (1988).

20. Reynolds, O. "An experimental investigation of the circumstances which determine whether the motion of water shall be direct or sinuous and of the law of resistance in parallel channels", Philosoph. Trans. $R$. Soc. Lond., 174, pp. 935-982 (1883).

21. Terashima, H., Kojima, S., and Homma, M. "Flagellar motility in bacteria structure and function of flagellar motor", International Review of Cell and Molecular Biology, 270, pp. 39-85 (2008).

22. Shi, L., Guo, S., and Asaka, K. "A novel jellyfish-like biomimetic microrobot", IEEEI/ICME International Conference on Complex Medical Engineering, pp. 277281 (2010).

23. Jayender, J., Patel, R.V., and Nikumb, S. "Robotassisted Active catheter insertion: Algorithms and experiments", The International Journal of Robotics Research, 28(9), pp. 1101-1117 (2009).

24. Kim, B., Lee, S., Park, J.H., and Park, J.O. "Design and fabrication of a locomotive mechanism for capsule-type endoscopes using shape memory alloys", IEEE/ASME Transactions on Mechatronics, 10, pp. 77-86 (2005). 
25. Langbein, S. and Czechowicz, A. "Adaptive resetting of SMA actuators", Journal of Intelligent Material Systems and Structures, 23(2), pp. 127-134 (2012).

26. Kim, B., Lee, M.G., Lee, Y.P., Kim, Y., and Lee, G. "An earth worm like micro robot using shape memory alloy actuator", Sensors and Actuators A: Physical, 125(2), pp. 429-437 (2006).

27. Apalkov, A., Fernández, R., Fontaine, J.G., Akinfiev, T., and Armad, M. "Mechanical actuator for biomimetic propulsion and the effect of the caudal fin elasticity on the swimming performance", Sensors and Actuators A: Physical, 178, pp. 164-174 (2012).

28. Wang, Zh., Hang, G., Li, J., Wang, Y., and Xiao, K. "A micro-robot fish with embedded SMA wire actuated flexible biomimetic fin", Sensors and Actuators A: Physical, 144(2), pp. 354-360 (2008).

29. Georges, T., Brailovski, V., and Terriault, P. "Characterization and design of antagonistic shape memory alloy actuators", Smart Materials and Structures, 21(3), 8 pages (2012).

30. Rao, A. and Reddy, J.N. "Design of shape memory alloy (SMA) actuator", Computational Mechanics, Springer, ISBN 978-3-319-03188-0 (2015).

31. Lauga, E. "Bacterial hydrodynamics", Annu. Rev. Fluid Mech., 48, pp. 105-130 (2016).

32. Chwang, T. and $\mathrm{Wu}, \mathrm{T}$. "A note on the helical movement of microorganisms", Proceedings of Royal Society of London, B, 178, pp. 327-346 (1971).

\section{Appendix A}

\section{Hydrodynamic parameters}

In this appendix we present the value of the hydrodynamics parameters of the helical flagellum and body:

$$
\begin{aligned}
& P_{11}=b_{1}+a_{1}, \quad P_{12}=P_{21}=b_{2}=c_{1}, \\
& P_{22}=\left(c_{2}-m_{\text {tail }}\right)+a_{2} .
\end{aligned}
$$

Eqs. (A.2) to (A.4) are according to Ref. [6] and they are used in Eq. (27):

$$
\begin{aligned}
& a_{1}=\frac{16 \pi \mu a e^{3}}{\left[\left(1+e^{2}\right) E-2 e\right]}, \\
& a_{2}=\frac{32 \pi \mu a b^{2} e^{3}}{3\left[2 e-\left(1-e^{2}\right) E\right]},
\end{aligned}
$$

$$
E=\operatorname{Ln} \frac{1+e}{1-e}
$$

Eqs. (A.5) to (A.9) are according to Ref. [11,32] and they are used in Eq. (27):

$$
\begin{aligned}
& b_{1}=n \lambda \cos \beta\left(\xi_{n} \tan ^{2} \beta+\xi_{t}\right), \\
& b_{2}=h_{\text {helix }} n \lambda\left(\xi_{n}-\xi_{t}\right) \sin \beta, \\
& c_{1}=h_{\text {helix }} n \lambda\left(\xi_{n}-\xi_{t}\right) \sin \beta, \\
& c_{2}=h_{\text {helix }}^{2} n \lambda \cos \beta\left(\xi_{t} \tan ^{2} \beta+\xi_{n}\right), \\
& m_{\text {tail }}=4 \mu \pi n b^{2} \cos \beta .
\end{aligned}
$$

\section{Biographies}

Hassan Sayyaadi received the BSc and MSc degrees in Mechanical Engineering from Amirkabir University of Technology, Tehran, Iran in 1987 and 1990, respectively. He also received his $\mathrm{PhD}$ degree in Mechanical Engineering from Tokyo University, Tokyo, Japan 2001. He is currently a Professor in the School of Mechanical Engineering, Sharif University of Technology, Tehran, Iran. His areas of expertise are dynamic modelling and system identification, vibration, autonomous underwater vehicles and remotely operated vehicles, robotics and control, mechanisms, optimization methods (reinforcement learning, genetic algorithm, etc.), smart materials, and intelligent systems.

Shahnaz Bahmanyar received the BSc and MSc degrees in Marine Engineering from Amirkabir University of Technology, Tehran, Iran in 2011. She is currently PhD Student in the School of Mechanical Engineering at Sharif University of Technology, Tehran, Iran. Her main areas of interest are biomimetic micro/macro swimmer robots, kinematic and dynamic modelling of swimming robots, low Reynolds number swimmer, biomimetic fish robot, hydrodynamic and maneuvering of marine vehicles. 